\title{
Uruguayan Cinema Today: Interviews with Three Uruguayan Directors
}

William Brown, University of Roehampton

María Soledad Montañez University of London

David Martin-Jones, University of Glasgow

\begin{abstract}
:
This article includes interviews with three contemporary Uruguayan film directors, Guillermo Casanova, Federico Veiroj and Silvana Camors. They answer questions about the state of cinema in contemporary Uruguay, offering varying perspectives on different aspects of Uruguayan film culture, from issues surrounding funding and distribution to the role of digital technology and the importance of maintaining film archives. Although the filmmakers identify numerous difficulties for Uruguayan filmmakers, especially at the level of institutional and financial support, they nonetheless remain grimly optimistic that Uruguayan cinema has a future.
\end{abstract}

\section{Keywords:}

Guillermo Casanova

Federico Veiroj

Silvana Camors

Uruguayan cinema

Film funding

Film archives

Below are interviews with three Uruguayan directors, each belonging in various respects to different generations - even if all three continue to be active today, both in Uruguay and 
elsewhere. Born in 1963, Guillermo Casanova belongs to a generation that came of age around the end of the dictatorship in Uruguay in 1985 - even if his career took off as an editor and director in the 1990s, and even if his most prominent films emerged in the new millennium, notably El viaje hacia el mar/Seawards Journey (2003) and Otra historia del mundo/Another Story of the World (2017). Federico Veiroj, meanwhile, was born in 1976 and belongs more clearly to the generation of filmmakers who saw Uruguayan cinema gain a global presence during the 2000s and the early 2010s, in particular through his feature films Acné (2008), La vida útil/A Useful Life (2010) and El apóstata/The Apostate (2015) - with a new film, Belmonte (2018), enjoying an initial run at international film festivals at the time of writing. Finally, while Silvana Camors is only marginally younger than Veiroj (she was born in 1978), she only latterly moved into audiovisual work after being trained as an artist. A member of the Laboratorio de Cine FAC in Montevideo, she has made a series of short experimental films throughout the 2010s, including Equisse (2016), Día 16/Day 16 (2016) and Desde aqui/From Here (2017), which are among numerous of her works curated by the Film-Makers' Cooperative in New York.

As a result of these 'generational' differences, and the different ways in which the directors work, we have been able to gather here some varied thoughts and opinions on diverse aspects of Uruguayan cinema, even if our questions return most consistently to the perceived state of the contemporary Uruguayan film industry, including the vexed question as to whether it exists at all. We are glad to report that the responses are candid, even if not always wholly optimistic in relation to the opportunities of and prospects for filmmaking in Uruguay.

That said, the directors often offer up contrasting opinions - with Casanova, for example, suggesting that first-time filmmakers have equal opportunities to secure funding as experienced directors, while Camors suggests the opposite. Furthermore, Camors feels that 
the preservation of Uruguayan cinema in archives has become a more prominent issue in recent times, while both Casanova and Veiroj disagree with this point of view.

Although we present the interviews to readers as if they were conducted in a simultaneous 'round-table' format, as we would have ideally liked, this was not actually possible on this occasion. Accordingly, when the speakers do disagree, we were not able to put them into dialogue with each other in order to see whether a consensus or reconsideration of such issues as funding for first-time filmmakers and audiovisual preservation might have been achieved. Nevertheless, we present the responses together, with their order varying according to how the interviewers felt best that the thoughts of the filmmakers informed and flowed into each other. In this way, it is possible to gain a more holistic insight into conditions facing filmmakers in Uruguay today.

All of the interviews were conducted in Spanish and so both the questions and the responses are reproduced here in their original language.

Studies in Spanish and Latin American Cinemas (SSLAC): ¿Cómo describirían la trayectoria de su carrera cinematográfica hasta ahora?

Federico Veiroj (FV): No pienso en trayectoria sino en cada película individual. La respuesta de la 'descripción' que me hace creo está en cada película; hablar de las películas es secundario cuando están para verlas.

SSLAC: Desde su punto de vista, ¿cuál es el estado actual de la industria cinematográfica en Uruguay?

Guillermo Casanova (GC): Agónico. 
FV: No existe una industria ni mercado. Mencionar industria es muchas veces un error importante en nuestro pequeño e ingenuo país donde la cultura es secundaria.

[Insert Figure 01. Caption: Federico Veiroj: 'no existe una industria ni mercado.']

Silvana Camors (SC): A mi alrededor percibo que en los últimos años ha crecido la realización de películas y éstas tienen cierto acceso a fondos públicos, a co-producciones en la región y participación en festivales, según sea su enfoque. Si bien se siente cierta desaceleración, no se ha interrumpido la realización de largometrajes de ficción y documental.

GC: Seguimos produciendo cine con la misma cantidad de dinero que hace 15 años, con un crecimiento absurdo de universidades que largan cada vez más realizadores y técnicos audiovisuales, donde en los concursos nacionales hay cada vez más participantes peleándonos por la misma cantidad de premios de hace años (donde allí nos encontramos todos, veteranos con experiencia y jóvenes debutantes que caen en la misma bolsa).

SSLAC: ¿Cuáles son los desafíos con los que se enfrentan, y qué oportunidades existen para la realización de la producción audiovisual?

GC: El desafío es que en Uruguay siempre se comienza de cero y las oportunidades son siempre las mismas. No hay nuevas atracciones que atrapen nuevos intereses. Más bien que la expectativa y aspiraciones cada vez son más reducidas. 
SC: El principal desafío que identifico se vincula directamente con el tipo de cine que realizo. Me interesa el cortometraje como pieza en si misma. A su vez trabajo con la imagen en movimiento en diferentes espacios, sea a modo de instalación en interacción con las imágenes en movimiento. Yo creo que son espacios donde los cineastas podemos desarrollar nuestro trabajo, sin embargo en Montevideo no hay demasiados fondos para su desarrollo. En cuanto a las oportunidades entiendo que cuanto más se filme en todas las áreas habrá más posibilidades para desarrollar mayor variedad de modos de hacer cine.

FV: El desafío es seguir haciendo películas y encontrar dinero para hacerlas, en el país que sea. En Uruguay no hay dinero para desarrollarse en cine, así que las oportunidades están fuera de fronteras.

SSLAC: ¿Cuál es la situación actual con respecto a financiación?

SC: Existen fondos públicos llamados por concurso pero son escasos. Imagino que ciertas películas pueden conseguir financiación privada o encontrar coproducciones con la región.

FV: Hay escasos fondos para el desarrollo de un cineasta. El problema es que no existe una manera simple y eficaz de incentivar a empresas privadas para que apoyen la cultura, el cine. Porque en un país del tercer mundo como Uruguay, que se financien películas (por más que sean cifras muy menores para lo que vale una película) ya es un milagro.

SSLAC: ¿Qué tan fácil o difícil es conseguir fondos para la producción audiovisual? 
SC: En mi caso aún no logro encontrar financiación. Los cortometrajes los realizo con las herramientas que tengo a mano y cubro casi la totalidad de los roles, intento que su costo de producción sea mínimo. En los proyectos que involucran imagen en movimiento en el espacio generalmente encuentro financiación dentro de fondos públicos de artes visuales.

FV: No hay problemas en conseguir fondos; hay simplemente desafíos como usted decía antes. Si no consigo dinero, me endeudo con privados y devuelvo más tarde. Hasta ahora ese modelo me ha funcionado.

SSLAC: ¿Creen que a un/a nuevo/a director/a le costaría más conseguir fondos?

FV: No lo sé.

GC: No, creo que no. Por ejemplo, en el último concurso del ICAU, del cual fui jurado, de los tres premios más importantes, cuatro finalistas eran óperas primas, y dos fueron ganadores.

SC: Sí. Hay pocos espacios para conseguir fondos y la trayectoria de los directores/as cuenta al momento de obtenerlos.

SSLAC: ¿Qué papel creen ustedes que desempeñan las nuevas tecnologías, como las cámaras digitales, en el desarrollo de la producción audiovisual en Uruguay?

FV: No lo sé, habría que mirar en los catálogos... Disculpas pero no tengo información para contestar esa pregunta. 
GC: Es una respuesta dificil de contestar en pocas líneas. Por un lado se abarata en luces, cámara y posproducción, pero por otro, los salarios de los técnicos y el dinero que se gasta en producción sigue siendo cada vez más caro.

SC: Creo que las nuevas tecnologías siempre han jugado un buen papel en el desarrollo de las artes, tanto en Uruguay como en el mundo. Al incorporarse nuevas herramientas para la creación de imagen en movimiento aumentan las posibilidades de los creadores, asimismo dentro de las nuevas tecnologías existen varias a bajo costo y fácil manejo, lo que posibilita el acceso a más cantidad de personas interesadas.

SSLAC: ¿Cuál es el rol que juega la televisión en la actualidad, tanto en términos de financiación, oportunidades, etc?

FV: CERO. En Uruguay la TV es importada de Argentina, Brasil, y lo local es vergonzoso. No hay ningún tipo de apoyo ni oportunidad. La TV es el enemigo del cine en Uruguay.

GC: Salvo el canal estatal, TNU, que tiene un premio para miniseries de 10 capítulos que no alcanza para otra cosa que una miniserie rodada en una casa en 30 días, no existe otra cosa. La ley de medios audiovisuales que salió en el gobierno de [José] Mujica fue un tiro en el pie para la ficción uruguaya, ya que el árticulo que obligaba a poner tres horas de ficción nacional, producido por los canales, en horario central, fue declarado inconstitucional por el tribunal supremo de la justicia uruguaya. Eso hizo que retrocediéramos varias décadas para atrás, ya que para lo único que están obligados es para producir productos de cualquier 
contenido, entrando allí los informativos, magazines o producciones de 'preguntas y respuestas.'

SC: Creo que tiene un papel muy menor. La televisión casi no genera contenidos fuera de lo periodístico, noticieros o programas de variedades. Hace unos años se crearon fondos específicos para la creación de series pero no conozco su funcionamiento.

SSLAC: ¿Qué tan útil o importante son para ustedes las formaciones/producciones transnacionales en su realización audiovisual?

GC: Es casi imposible hacer una película sin que no se consiga una coproducción internacional.

SC: Al ser un país chico con poca historia cinematográfica creo que mirar al mundo es muy importante. Es sumamente importante poder formarse fuera de Uruguay y enriquecedor formar equipos de trabajo con personas de otros lados.

FV: Financiera y creativamente todos los proyectos que he hecho hasta ahora están llevado adelante con personas o fondos de varios países. Fundamental para las películas que me tocaron hacer hasta ahora.

SC: El cine es como la droga, la primera vez es mucho más barata que la segunda (sobre todo por que la primera vez casi siempre se produce de forma cooperativa y por propio impulso de sus realizadores, director y productor). Producir una película uruguaya es una carrera de obstáculos. Una vez que se consigue un premio ya quedás comprometido a su realización. A 
partir de allí para lograr llegar a la meta, debés de ganar todos los concursos nacionales y de coproducciones internacionales, que son casi siempre con Argentina y/o Brasil. O sea, que para lograr llegar a la sala o se gana todos los premios o la película queda media enclenque. Eso hace que tu guión ha de gustarles a todos los jurados de todos los concursos. Por lo tanto muchas veces se hace la película que quieren los otros. Son los gustos de otros, entonces, los que nos dejarían hacer.

[Insert Figure 02. Caption: Silvana Camors: 'El cine es como la droga.']

SSLAC: En términos de reconocimiento del cine nacional en los festivales de cine internacionales, ¿creen ustedes que ha habido una caída en tal reconocimiento?, y si es así, ¿ha generado alguna diferencia en la industria en Uruguay?

GC: No lo sé a ciencia cierta, lo que sí es cierto que si los directores que han obtenido reconocimiento internacional se les dificulta tener una continuidad en sus realizaciones es muy dificil mantener ese impulso inicial como el que tuvo el cine uruguayo en la primera década del 2000.

SC: No sé si hubo una caída, aparentemente el reconocimiento internacional hace que desde acá se preste mayor atención a las producciones locales. En ese sentido podría ser que si hay menos reconocimiento en festivales internacionales, las películas tengan menor atención del público. Pero no estoy segura que sea así.

FV: Creo que esto es materia para estudiosos del cine con la nacionalidad uruguaya. Le hablo de mi caso que es el que conozco: todas mis películas estuvieron en festivales 
internacionales: Toronto, San Sebastián, Cannes, Mar del Plata, y la última llamada Belmonte la estrené este mes en Toronto, y en unos días en San Sebastián.

SSLAC: ¿Es la distribución un aspecto fundamental para el cine uruguayo, tanto a nivel nacional como internacional? Es decir, desde su punto de vista, ¿qué es posible hacer y qué se podría mejorar?

FV: Domésticamente el mercado es casi nulo, al menos para las películas que yo he realizado. Yo trabajo con el mundo entero, sea con ventas independientes a países o ventas regionales, o worldwide.

GC: No hay uruguayos dedicados a la distribución internacional de la producción uruguaya, salvo el trabajo individual del productor de cada película de buscar distribuidores internacionales extra muros. Tampoco hay una política seria a nivel estatal al respecto.

SC: Entiendo que la distribución es fundamental para que las películas se vean, pero no conozco bien el funcionamiento comercial de esa área para dar una opinión al respecto.

SSLAC: ¿Qué rol juega la preservación/restauración de archivos audiovisuales en la cultura uruguaya?

GC: Diría que casi nula, hay un mínimo mantenimiento de archivo antiguo, $35 \mathrm{~mm}$ del siglo XX y pasaje de videos analógicos al digital de materiales de los finales de la década del 80 y 90, pero no hay ninguna política de preservación y archivo del cine uruguayo del siglo XXI. 
No hay una forma de archivar y lograr poder ver los materiales producidos posteriores a la muerte del DVD.

SC: Siempre es importante conocer y tener acceso a las obras que se han generado a lo largo del tiempo, que se puedan ver, analizar y reflexionar en el contexto general. Creo que en los últimos años se ha puesto mas atención a los archivos y se han generado debates sobre preservación y restauración. Al ser un área que requiere fondos para su desarrollo creo que el proceso es lento si no se cuenta con apoyo económico.

FV: En Uruguay hay enorme ignorancia y desidia en todo lo relacionado a archivos. La gente detrás de los organismos privados y públicos que se dedican a esas tareas es inoperante. Se precisa mucho dinero que en un país del tercer mundo como Uruguay nunca se va a lograr; es un partido que nunca se ha jugado y ni se jugará ahora con la escasa tradición de cine producido localmente.

SSLAC: ¿Creen ustedes que hay algún cambio en la producción audiovisual debido al acceso a los archivos de cine, o la disponibilidad y acceso a películas internacionales, video-ensayos, etc?

SC: En las producciones uruguayas no siento que se haya incorporado estos materiales a sus producciones. No veo obras que enfoquen su trabajo en archivos, ni fílmicos, ni digitales, ni de acceso libre online. Hay algunos casos puntuales pero los veo como una inquietud puntual del director en esa obra. El mayor uso que veo es en los documentales que incorporan el archivo para ilustrar acontecimientos pasados. 
FV: No entiendo la pregunta la verdad... Yo miro todo tipo de cine, y me gustan los archivos. Procedo de trabajar en cinematecas y hago películas porque no he encontrado mi sitio aún en un archivo en condiciones donde trabajar en programación, restauración, conservación, y divulgación del cine de todo el mundo y de todas las épocas. Me gusta más pensar en todo el cine que en lo que hago personalmente que es algo que me fascina también y es el motor de mi vida laboral. Ambiciono un trabajo así.

SSLAC: ¿Opinan que las representaciones más aproximadas o interesantes de Uruguay son las que se ven los cines o quizás en internet? ¿Por qué?

FV: No tengo idea... Y naturalmente lo que a alguien le parece interesante y auténtico, a otro le puede resultar tedioso y repetido.

SC: Siento que aún la proyección en sala de cine le da un prestigio a la obra que internet aún no tiene. En este sentido creo que los festivales y muestras juegan un rol interesante y sería bueno pensar como hacer para que funcione la distribución por internet.

GC: Hay un cambio en la costumbre de los espectadores de cine. El cine hoy está enfocado al cine juvenil, de súper héroes, de terror y de 'rápidos y furiosos/fast \& furious.' Hoy el joven es el que sale de la casa de sus padres para ir a ver cine. Los padres se quedan en sus casas para ver cine por streaming. Por ello se hace necesario que exista una política de estado con respecto al streaming nacional, como sucede en varios países europeos o Argentina. También debería de crearse, como en el caso de Brasil, salas digitales para la exhibición de producciones nacionales. En São Paulo existen 50 nuevas salas para tales casos. En Uruguay no existe una sola. 
SSLAC: El preguntón: ¿existe el cine uruguayo?

SC: Hay cine en Uruguay. Supongo que las ideas de un cine uruguayo se construyen desde miradas lejanas, estando dentro no sé si puedo definirlo así. En Uruguay se está filmando, sería lindo que cada día se filme más y que generemos mucho material para que haya muchas personas pensando sobre si existe el cine uruguayo

FV: Sí, claro, hay películas uruguayas. Eso está genial que exista, pero no por la nacionalidad sino porque hay gente que quiere hacer cine y utilizar el tan hermoso y potente medio para contar historias. Siempre debería existir si se pudiese, es un privilegio enorme que existan las películas de cualquier lado del mundo, un placer poder ver películas buenas o al gusto de cada uno.

GC: Sí, creo que sí, en la medida que se sigue pensando en producciones con un contenido idiosincráticamente uruguayo, contando su manera de ser y de su decir. Las nuevas generaciones siguen en la búsqueda identitaria, como pueden ser las futuras películas de Pablo Abdala y Joaquín Peñagaricano, Mateina, Lucía Garibaldi, Los Tiburones y La Última Reina o Rafael y Bernardo Antonaccio, Matarifes.

SSLAC: Y pensando en el futuro, ¿qué les facilitaría como director seguir realizando películas en Uruguay?

GC: Uno debería preguntarse qué ha pasado con todos los directores que han dirigido cine en Uruguay y viviendo en Uruguay que hayan tenido éxito y preguntarse porque no han podido 
dirigir películas en una continuidad razonable. Muchos han quedado con una, Pablo Dotta, Enrique Fernández. Pablo Stoll no saca una película uruguaya desde 2009 al igual que Beatriz Flores Silva. Yo he demorado 14 años entre El viaje hacia el mar y Otra Historia del Mundo. Uno está casi siempre con un pie en el abandono.

[Insert Figure 03. Caption: Guillermo Casanova: 'Uno está casi siempre con un pie en el abandono.']

SC: Que existan fondos que amplíen su alcance a todas las maneras de hacer cine, que existan más lugares de exhibición alternativa, que existan más centros de formación que generen más personas interesadas en hacer y otros centros de formación que amplifiquen diferentes modos de ver, que en los centros de formación inicial (escuela primaria y secundaria) se promueva ver imagen en movimiento más allá de las pantallas tradicionales.

FV: Que las empresas privadas tengan una forma simple y directa de invertir en cine. El apoyo público con los nefastos políticos de turno, y sobre todo de la cultura, es una batalla perdida; aunque de todos modos, no pienso que el cine debe estar estrictamente financiado por fondos públicos en países pobres como Uruguay.

GC: Creo que la base está en cambiar en la forma de conseguir fondos para la financiación y que sería, al igual que en Argentina, financiación por ventanilla, es decir, conseguir financiación por etapas y por historial. En Uruguay siempre es arrancar de cero, sin importar la carga acumulada que lleva. 
SSLAC: ¿Cuál les parece sería la dirección que el cine uruguayo debería tomar? Por ejemplo, ¿creen que la industria se debería enfocar en hacer más o ‘mejores’ películas?

GC: Hace una década atrás se hacían cuatro o cinco películas por año, y con ellas obteníamos reconocimiento por parte del público, de la crítica y de los festivales internacionales. Hoy seguimos produciendo la misma cantidad pero sin las gratificaciones obtenidas en el pasado. Hoy, a pesar de que existen más políticas de estado, estamos igual que antes que obtuviéramos todos los premios y reconocimientos nacionales e internacionales cuando solo existía un solo fondo nacional de cine (el FONA).

FV: No hay industria uruguaya... Yo pienso en calidad, pero eso no depende de un país o de más o menos apoyo, depende del talento que haya detrás de las películas. Eso es lo que hay que proteger más que nada; algo imposible que suceda aquí, claramente, ya que somos un país donde ni siquiera los maestros o profesores tienen buenos sueldos... ¡Imagínese!

GC: Ni productores ni realizadores en Uruguay han vivido del cine. Digamos que los que lo lograron en todo caso han sobrevivido hasta lograr realizar el siguiente proyecto. No hay un mercado nacional para el cine comercial. Para hacer cine comercial hay que salir del país, como lo hizo Fede Álvarez o quedarse en el país que lo acogió como en el caso de Álvaro Brechner. Es muy simple, para poder hacer cine nacional se requiere de políticas de estado serias, como hoy puede ser el caso de Colombia.

SC: Habría que definir que es 'mejores películas,' las que vendan muchas entradas si se entrenan en cine, que tengan muchas visitas si te opta por la vía online, que sean consideradas por expertos como obras de arte, que críticos de cine les den muchas estrellas, que en las 
escuelas de cine y universidades las estudien, que quienes las hacen se sientan satisfechos con la obra generada. Al no poder responder sobre qué es una mejor película, creo que se debería filmar mucho y ver que va pasando.

GC: Siguiendo la premisa de que aún no hay una industria cinematográfica, como sí la hay, y muy fuerte, en la industria audiovisual publicitaria y que muy poca gente puede vivir del cine (y ellos son en su mayoría técnicos de la jerarquía media, asistentes de dirección, de producción y de arte, sonidistas y editores), el que logra llegar hacer una película nacional, ya debería ser premiado solo por haberlo logrado. El grave problema es que siempre será una película refritada por años en la cabeza de su realizador y cuando llega a la sala ya es una película vieja desde su concepción.

FV: El camino del cine uruguayo es uno de talento, pasión, ganas de contar historias. Contra eso no hay lucha que se pueda perder.

GC: Con un grupete de amigos del cine, cuando por ejemplo son las cuatro de la mañana de un día de invierno en exteriores y estamos filmando la última toma, decimos que andamos terminando el penúltimo capítulo del libro... ¿Qué libro? pregunta un despistado. El Cine Nacional y la Reputa Madre que lo Parió.

\section{Contributors' Details}

William Brown is a Senior Lecturer in Film at the University of Roehampton, London. He is the author of Non-Cinema: Global Digital Filmmaking and the Multitude (Bloomsbury, 2018) and Supercinema: Film-Philosophy for the Digital Age (Berghahn, 2013). He also is a maker of zero-budget films including En Attendant Godard (2009), Common Ground (2012), 
Selfie (2014), Circle/Line (2017), and This is Cinema (forthcoming). He is also currently seeking publication for his book, Kinoteuthis Infernalis: The Emergence of Chthulumedia, which is co-written with David H. Fleming, and which deals with cephalopods and tentacles in cinema and new media.

Queen's Building, Rm QB018, Department of Media, Culture and Language, University of Roehampton, London, SW15 5SL

E-mail: William.Brown@roehampton.ac.uk

Orcid iD: 0000-0002-9367-6418;

David Martin-Jones is Professor of Film Studies, University of Glasgow, UK. His research engages with what it means to study a world of cinemas, exploring key contemporary issues (e.g. time, ethics, transnational identities, world history, ecology, the Anthropocene) to unlock film's engagement with globalization. He is the author/editor of eight books, including Cinema Against Doublethink (2018). He has published in numerous journals (including Cinema Journal, Screen and Third Text), co-edits the Bloomsbury monograph series Thinking Cinema, and serves on the editorial boards of Film-Philosophy and Deleuze and Guattari Studies.

Contact:

E-mail: David.Martin-Jones@glasgow.ac.uk;

María Soledad Montañez is a Research Fellow in Community Engagement at the Institute of Modern Languages Research, University of London. Her interests range across diverse areas of Latin American literature and cinema, with a specific focus on gender, memory and national identity. She has published several articles on Uruguayan cinema in Studies in Hispanic Cinemas (2007), Screen (2009), Latin American Perspectives (2012). In the last few years, she became more interested in community work and has engaged in community 
projects with the Spanish and Latin American communities in London and Glasgow, bringing and translating her academic skills into the voluntary sector.

Contact: Film and Television Studies, University of Glasgow, Gilmorehill Halls, 9 University Avenue, Glasgow, G12 8QQ.

E-mail: mariasmontanez@gmail.com;

Silvana Camors is a filmmaker who graduated from Escuela de Cine del Uruguay and who has made several shorts and installations combining digital and analogue filmmaking. She is interested in the exploration of light in open spaces, the relationship between the still image and the moving image, and their interaction with the human body. She has participated in national and international film festivals, and coordinates and teaches courses on experimental cinema and expanded cinema at Uruguay Campus Film. She also teaches workshops on collective film production and stop motion for children. Her Vimeo channel is

\section{https://vimeo.com/silvanacamors;}

Guillermo Casanova was born in Montevideo, Uruguay in 1963, and is a founding member of the film production Centro de Medios Audiovisuales (CEMA). He has directed the documentary short Mamá era punk/Mama was a Punk(1988) and the feature films El viaje hacia el mar/Seawards Journey (2003) and Otra historia del mundo/Another Story of the World (2017). He is also founder and co-director of Lavorágine Films, which has produced films such as Ruido by Marcelo Bertalmío, Flacas vacas by Santiago Svirsky, and Enrique Buchichio's Zanahoria. Casanova's films have received numerous awards at international film festivals;

Federico Veiroj was born in Montevideo, Uruguay, in 1976. He is a director and producer, known for Acné (2008), La Vida Útil/A Useful Life (2010), El apóstata/The Apostate (2015) and Belmonte (2018). He also worked as a script supervisor on both Whisky (2004) and 25 Watts (2001), in which he also featured as an actor. His films have played at numerous film 
festivals around the world, having also won many awards, including at the AFI Fest, the Havana Film Festival, the Istanbul International Film Festival, the Mar del Plata Film Festival, the San Sebastián International Film Festival and the Warsaw International Film Festival. 